\section{La disciplina que estudia las relaciones entre la lengua y la sociedad: sociolingüística}

The discipline that studies the relations between language and society: sociolinguistics
Francis Mendoza Morán Universidad Nacional Autónoma de Nicaragua. menmoran@hotmail.com

(c) UNAN-Managua Recibido: abril 2018 Aprobado: junio 2018

\section{RESUMEN}

Este breve análisis expone una panorámica general sobre sociolingüística, destacando su desarrollo histórico e importancia actual. También resalta la función de algunas variables sociales en los estudios del lenguaje.

Palabras clave: sociolingüística, étnico, nivel de instrucción, género, clase social.

\section{ABSTRACT}

This brief analysis exposes an overview on sociolinguistics, emphasizing their historical development and current importance. It also highlights the role of some social variables in language studies.

Keywords: sociolinguistic, ethnic, educational level, gender, social class.

A través del tiempo, la sociolingüística ha recorrido caminos con muchas dificultades y con puntos de vistas divergentes. El término 'sociolingüística' ha sido muy discutido por diferentes estudiosos, uno de ellos es López (1989) quien dicta que:

Desde 1952, cuando aparece por primera vez el término sociolingüística en el título de un trabajo (Currie, 1952), hasta la fecha, suman varios centenares de libros, artículos, comunicaciones a congresos y simposio, capítulos para volúmenes colectivos, reseñas y notas que aparecen encabezados con esta o semejante designación. (p. 7).

En medio de esas controversias conceptuales, la sociolingüística brinda la oportunidad de analizar diferentes fenómenos del acto de habla. Dubois (1994) coincide con López (1989) cuando afirma que:

La sociolingüística puede tomar en consideración como dato social el estado del emisor (origen étnico, profesión, nivel de vida, etc.) y vincular a este estado el modelo de actuación obtenido. Es evidente que, así definida, la sociolingüística engloba prácticamente toda la lingüística que procede a partir de un corpus ya que estos siempre se producen en un tiempo, lugar y medio determinado. (p.577).

Por lo tanto, se puede asegurar que esta ciencia inicia un cambio en el análisis de diversas variantes dialectales de una comunidad de hablantes determinada. Y es por eso que hablamos de esa relación que tiene la sociolingüística con la etnolingüística, la sociología, la geografía lingüística y la dialectología.

No hay que dejar atrás el comportamiento de la conducta verbal que es considerada una conducta social; no es un mero medio de comunicación sino transmisión de significados sociales. 
Esa es una de las razones por las cuales se busca una respuesta al estudio del análisis oral y su vinculación con el contexto social donde se desarrolla un acto comunicativo. Al respecto Weinerman (1976) considera que:

Durante largo tiempo, sociólogos y psicólogos han ignorado este tipo de conducta como si no fuera un fenómeno de su competencia y solo pertinente a los lingüistas. La situación no era muy diferente entre los lingüistas, quienes estaban ocupados en el estudio de los elementos universales de la lengua (con exclusión de la diversidad del habla), en el que las variables sociológicas y psicológicas no tenían lugar. Pero las primeras décadas de este siglo fueron testigos de un importante cambio. (p.17).

En medio del desarrollo de la sociolingüística se revelan conceptos como el de competencia lingüística y competencia comunicativa que inciden en la comunidad (lingüística). Cada "comunidad" poseerá reglas que serán compartidas en relación al fenómeno de habla. Es importante subrayar lo señalado por Hymes (1962), citado por Weinerman, (1976) cuando asegura que: "El interés del campo de la sociolingüística consiste en el estudio de la conducta lingüística (y de todas las otras conductas que usan alternativas de los signos lingüísticos), en términos de las relaciones entre los componentes del acto de habla. (p. 22).

De manera que los dos significados de cualquier emisión verbal, significado referencial y social, están sujetos a dos clases diferentes de reglas: lingüísticas y comunicativas (o sociolingüísticas). Esta idea se reafirma en el planteamiento brindado por Dubois (1994), cuando enfatiza en que, es la sociolingüística la que considera como dato social el momento del que codifica un mensaje, entre estos se razona sobre su origen étnico, profesión, nivel de vida, etc. Este mismo autor asegura que: "La sociolingüística tiene como tarea mostrar, en la medida de lo posible, la covariación de los fenómenos lingüísticos y sociales y, eventualmente, establecer una relación de causa a efecto". (p. 577).

\section{Desarrollo de la sociolingüística}

¿Cómo se inicia la sociolingüística? Como toda ciencia nos presenta una historia. Y la complejidad que se muestra sobre su concepto, es muy amplia. Por eso es que algunas concepciones son equívocas. A criterio de López (1989) el término (sociolingüística) aparece por primera vez en una publicación (Currie, 1952). Determina que en algunos lugares se ha desarrollado el enfoque de esta, tales como los Estados Unidos, Canadá e Inglaterra. A partir de 1964 se inicia una gran tarea por avanzar en estos criterios, dentro de estos debemos mencionar a William Wright. Este es quien realiza una reunión en Los Ángeles con doce especialistas con el fin de revisar los objetivos de esta disciplina. Posteriormente surgen los nombres de grandes figuras de la teoría y la investigación: William Labov, Henrietta J. Cedergren, David Sankoff, Peter Trudgill, R. A. Hudson, Michael Halliday. Joshua A. Fishman, Jonh J. Gumperz, Dell H. Hymes y Charles A. Ferguson. Los datos que nos ofrece López (1989) coinciden con los de Conde (2005) al asegurar que: "La sociolingüística comienza a desarrollarse en la década de 1960-70, gracias a la conjunción de una serie de factores". (p.23).

En el ámbito hispano la sociolingüística fue despaciosa y demorada. López (1989), menciona como pionero el trabajo de Manuel Alvar con su texto titulado Niveles socio-culturales en el habla de Las Palmas de Gran Canaria, que es de 1972. Después sobresalen otros como antologías de artículos. Es donde surge la realizada por Paul L. Garvin y Yolanda Lastra, Antología de estudios de etno-lingüística y sociolingüística, que se produjo en México en 1974. 
Le siguió la de Óscar Uribe Villegas, La sociolingüística actual: algunos de sus problemas, planteamientos y soluciones. Ambas mexicanas y con poca suerte. Seguidamente nace la elaborada por Francisco Abad, Lecturas de sociolingüística, Madrid, 1978. Después está Unidades de sociolingüística, escrita por Humberto López Morales, 1978.

Sin embargo, hasta ese momento los diferentes puntos de vista dejaban entrever ciertas dudas con relación a la caracterización en general de esta disciplina. Luego nacen otras publicaciones, tal es la de 1989 por Carmen Silva Corvalán (chilena, radicada en Estados Unidos) denominada Sociolingüística. Teoría y análisis. Le sigue la obra de Karmele Rotaetxe, Sociolingüística. López (enfatiza) en que estos estudios son el reflejo de un gran esfuerzo y que expresan los puntos de vista de los autores para iniciar al público español en este tipo de concepciones. Igual ocurre con el texto de 1989 en catalán de Francisco Gimeno y Brauli Montoya, titulado Sociolingüística.

Después se desarrolló una serie de traducciones que inició con la Soziolinguistik, Eine Einfuhrung de Brigitte Schlieben-Lange en 1977. Mientras tanto, en 1978 se traduce la Intriduction á la Socioliguistique de Jean Baptiste Marsellesi y Bernard Gardin. Morales (1989) describe que en este texto se dedican breves páginas a Sapir y Whorf y se describe la historia de la lingüística. Meses después, 1980, se traduce The Sociology of Language de Joshua A. Fishman. En 1982, Sociolinguistics de R. A. Hudson, que es un buen material introductorio. $Y$ por último en 1983, Sociolinguistic Pattenrs de William Labov. Posteriormente con el libro de Francisco Moreno, Sociolingüística en E.E. U.U. 1975-1985. Guía bibliográfica crítica (1988), las publicaciones en España sobre sociolingüística fueron superadas.

Subsiguientemente en (Hispanoamérica) México brotaron libros como el de Óscar Uribe Villegas: Sociolingüística concreta (algunas facetas) y Sociolingüística: una introducción a su estudio, los dos en 1970. En 1971, Sociolingüística doctrinaria. Luego en 1972, Situaciones de multilingüismo en el mundo y Las disciplinas sociolingüísticas y el énfasis sociológico en 1976. Mientras que en los textos Acercamiento histórico a la sociolingüística, de 1976, se revisa el origen de la ciencia y el estudio es enfocado desde la perspectiva antropológica e informativa y en Introducción a la teoría y práctica de la sociolingüística de 1982, se abordan muchos contenidos. Por otro lado, se hace notar la presencia de los Cuadernos de Lingüística de la Asociación de Lingüística de la América Latina (ALFAL), especialmente el número 3, En torno a la sociolingüística, 1978, y el número 5, Estudios de sociolingüística, 1978. Ahí se recogen trabajos de Lope Blanch, Manuel Alvar y Humberto López Morales.

El autor López (1989) establece que todas y cada una de esas publicaciones tienen su relevancia hacia la profundización de la sociolingüística, pero que tienen sus debilidades. No obstante, también presenta una reflexión hacia la importancia de las asociaciones lingüísticas que apenas por ahí, le brindan la importancia a esta ciencia. Por lo tanto, si no existe empuje en esa vía, no se debe esperar mayores resultados en los futuros trabajos investigativos. Y que a pesar de esos pocos y representativos textos, la situación poco a poco va mejorando, así tenemos las investigaciones más sobresalientes como las de López (1986), Lavandera (1981) y el de Vallverdu (1980). También los de Francisco Gimeno, Brauli Montoya, José Ramón Gómez Molina, Julio Borrego Nieto, Francisco Miguel Martínez Martín, Manuel Almeida, Maitena Etxebrría, Francisco José Zamora y tesis recientes. 
Como se observa, evidentemente hay un brío de la ciencia sociolingüística, sin embargo, Moreno (2008) nos aclara que: "La sociolingüística no surgió de la nada para explicar lo que nadie podía explicar, aunque hiciera suyos problemas presentados desde puntos de vista muy diferentes y quisiera darles una salida con unos modelos abiertos y coherentes y, sobre todo, con unas bases metodológicas sólidas". (p.288).

\section{Las variables sociales}

\section{El sexo}

De esta manera nos enfrentamos a analizar aspectos de las denominadas "variables sociales", así como también del estudio de las "actitudes lingüísticas". Blas (2008) afirma que: "La lengua representa una forma de conducta social de la que se desprenden con frecuencia diferencias muy significativas entre los hablantes" (p. 135). Por ende, la correspondencia entre sociedad y lengua, son aspectos inseparables. Y es que la historia ha demostrado de forma científica que ambos factores, sociales y situacionales-lingüísticos- actúan de manera probabilística sobre el estudio de la variación lingüística. Esa es una de las razones por las cuales la mayoría de los estudios sociolingüísticos se inclinan hacia la aplicación de una metodología estadística-cuantitativa que determine esa "probabilidad" del fenómeno a observar, en este caso es el nivel fonético.

Así se mencionan variables como: el contexto en que se realiza una determinada comunicación, las relaciones entre los que participan o características sociales y dentro de estos menciona a las adscritas: el grupo generacional, étnico, raza, sexo, edad, casta, etc. Y las adquiridas: nivel educacional, socioeconómico, etc.

Acorde a este planteamiento Moreno (2008) indica que: "La investigación sociolingüística ha permitido conocer que las variables sociales que influyen sobre la variación lingüística lo hacen de un modo específico en cada comunidad y respecto a fenómenos lingüísticos concretos". (p. 39). Por lo tanto, los diferentes factores sociales no pueden funcionar de igual manera en cada una de las comunidades. Una comunidad mayor tiende a presentar mayor complejidad social y conlleva a una superior variación lingüística, lo que indica que el uso de la lengua será más heterogéneo. Moreno (2008) y Blas (2008) coinciden en que los factores sociales que demuestran más influencia sobre la variación lingüística son: el sexo, la edad, el nivel de instrucción, el nivel sociocultural y la etnia.

La variable social referida al "sexo", según Moreno (2008), fue trabajada allá por el año 1952 con la publicación en la revista Orbis acerca de un trabajo que abordó la cuestión sobre la lengua de las mujeres, Le langage des femmes: Enquete linguistique à l'èchelle mondiale. Además, también describe que en esa época se discutieron temáticas como la relevancia de utilizar mujeres como informantes y la característica innovadora de la forma de su habla. Contrario a esto Alvar (1956), citado por Moreno (2008), parte de un tratado sobre el habla de Puebla de Don Fadrique, en Andalucía, y llega a conclusiones interesantes, asegurando que el arcaísmo o la innovación del habla de las mujeres no estriba en el sexo, según el tipo de vida de ese zona y que en cambio en el lugar de Andalucía, las mujeres presentaron rasgos mayoritariamente innovadores en comparación con los hombres, incluso llega a asegurar que: "Decir que el habla femenina es conservadora, neologista o ni una cosa u otra es, en verdad, no decir demasiado, por cuanto en su contexto social puede ser cada una de esas cosas o todas ellas, y fuera del ámbito al que pertenece no es nada". (p. 41). 
Por lo tanto, el sexo puede mostrarse más como un factor de segundo orden, subordinado a dimensiones sociales distintas y con mayor determinación. Una de las labores de la sociolingüística es observar la conducta lingüística de los hombres y las mujeres, esto ha sido y se ha mantenido como permanente punto de atención en diferentes estudios con enfoque sociolingüístico hasta nuestra actualidad, aunque se hagan aseveraciones inconsistentes como: las mujeres son conservadoras, inseguras, sensibles, solidarias y expresivas, mientras que la de los hombres es independiente, competitiva y jerárquica.

Las nuevas investigaciones sociolingüísticas se han encargado de apartar estas posturas, demostrándolos con fundamentos. Entre estos se subrayan los que establecen diferencias entre ambos sexos y que lo enfocan desde el punto de vista etnográfico, estos representan datos interesantes en cuanto a la conducta comunicativa tanto de los hombres como las mujeres. El autor, Moreno (2008), describe el de Saville-Troike con el trabajo The Ethnography of Comunication de 1982, este refiere varios ejemplos como el que las mujeres de algunos dialectos esquimales utilizan nasales sonoras en posición final [m, n, -ng], mientras que los hombres usan las oclusivas sordas [p, $t, k]$ y otros aspectos más.

En cuanto a las investigaciones sociolingüísticas de la zona urbana se manifiestan aspectos importantes, tal caso como el que las mujeres demuestran una actitud más positiva que los hombres sobre las utilizaciones que rigen por la norma, estos hallazgos se perfilan es estudios como el de Labov (1972) y hasta ha dado lugar a que se le denomine modelo sociolingüístico de sexo. Vinculado a esta concepción López (1992) afirma que: "En una estratificación sociolingüística estable, los hombres usan formas que no son estándares con mayor frecuencia que las mujeres, siempre que la variación se produzca en un nivel de consciencia dentro de la comunidad de habla". (p. 52).

Lo cierto es que se siguen originando grandes controversias en torno a esto, Blas (2008) alega que: "Pese al importante salto cualitativo aportado por la sociolingüística en el tratamiento de las diferencias entre hombres y mujeres, la dialectología ya se había ocupado de ellas desde hacía décadas". (p.158). Se deduce que ambos elementos, mujer y hombre, son hechos fundamentales para ser observados en cualquier acto de habla. Cada estudio obtiene respuestas diferentes, porque responden a objetivos diferentes, quizás algunos le den mayor importancia solo a las mujeres o solo a los hombres. Una gran verdad es que esto es digno de prestársele atención, especialmente al involucrarnos con sociedad y lingüística.

\section{La edad}

La variable social "edad" se considera uno de los factores sociales con más fuerza y claridad en los usos lingüísticos de una comunidad de hablantes. Moreno (2008) establece que la "edad" condiciona la variación lingüística con mayor predominio que otros factores. Contrario a la "clase social" o el "género", la "edad" es constante y en su realidad no se ve alterada por cambios socioeconómicos, actitudes u organización. Mientras el tiempo sigue su camino, la edad va estableciendo y cambiando los caracteres y los hábitos sociales de las personas, dentro de estos se encuentran los comunicativos y los puramente lingüísticos. Con relación a "edad", "sexo" o "género", Richard Cameron, citado por Moreno (2008), demostró que las diferencias lingüísticas basadas en el género varían en el transcurso de la vida y destaca como elemento esencial que el desarrollo lingüístico de una persona adquiere mayor importancia durante la etapa de adquisición del dialecto y del sociolecto. 
Se considera valioso el aporte de Willian Lavob en 1964, cuando establece una división continua de seis fases para la adquisición del inglés estándar en donde incluye las variedades regionales, sociales y estilísticas. Lavob define como sumario de aculturación o de alejamiento de los usos que se producen durante la adolescencia y adultez. Gráficamente es así:

\begin{tabular}{|c|l|l|}
\hline \multicolumn{3}{|c|}{ Etapas de adquisición del inglés estándar, según estudio de Lavob (1964) } \\
\hline$N^{\circ}$ & Aspecto & Edad \\
\hline 1 & Adquisición de la gramática básica & Primera infancia \\
\hline 2 & Adquisición del vernáculo & 5 y 12 años \\
\hline 3 & Desarrollo de la percepción social & 14 y 15 años \\
\hline 4 & Desarrollo de la variación estilística & 14 años aprox. \\
\hline 5 & Mantenimiento de un uso estándar & Primera etapa adulta \\
\hline 6 & $\begin{array}{l}\text { Adquisición de todos los recursos } \\
\text { lingüísticos }\end{array}$ & $\begin{array}{l}\text { Persona instruida y } \\
\text { preocupada por el uso }\end{array}$ \\
\hline
\end{tabular}

La explicación a esas posibilidades radican en que la primera fase se desarrolla bajo la dirección de los padres, especialmente de la madre o el familiar más cercano; la segunda bajo el dominio de amigos y compañeros de estudios; la tercera de los hablantes adultos; la cuarta necesita de relaciones sociales diferentes (familia, compañeros, vecinos). En esta clasificación, se interpreta que a partir de la segunda etapa se inicia el desarrollo post-vernáculo que agrega aspectos de variados medios. La propuesta de esta división ha dado origen a diversas controversias, no obstante esta es un marco de referencia apreciable y su discusión aún no concluye.

Al respecto de la variable social edad, Blass (2008) señala que: "Por lo que se refiere a la sociolingüística; una de las ideas más recurrentes en la bibliografía es que la edad representa un factor que puede condicionar la variación en un grado incluso mayor a como lo hacen otros parámetros sociales tan relevantes como el sexo o la clase social" (p.190). Es decir, que la edad forma parte esencial y es digna de observación, como de análisis en sus distintos indicadores.

Estas y otras manifestaciones del campo de la sociolingüística nos lleva de la mano a decidir sobre qué edad deberá considerarse para realizar una investigación. Al respecto, las respuestas han sido diferentes. Moreno (2008), destaca los tratados de P. Bentivoglio y M. Sedano (1993), quienes efectuaron en Caracas un estudio basado en hablantes de más de catorce años. También describe el de O. Alba, en Santiago de los Caballeros y el de Perissinotto, en México, ambos trabajan con edades de dieciséis años a más. Luego ubica el de López (1983), en San Juan de Puerto Rico. Todos ellos trabajan con hablantes mayores de veinte años. 
Cabe mencionar que en Nicaragua se ha seguido, en algunos casos, el modelo del Proyecto para el estudio sociolingüístico del español de España y de América (PRESEEA) que también determina trabajar con informantes a partir de veinte años.

Como se observa esta división obedece a los objetivos de esta tesis y además al punto de vista de Moreno (2008) quien asegura que: "Sea como sea, la sociolingüística no suele considerar conveniente la recogida de datos de hablantes menores de 14 o 15 años para el estudio de grandes núcleos urbanos, al menos que no esté suficientemente claro cómo y cuándo se llega a la madurez en el uso social de la lengua". (p.49).

\section{Clase social}

La variable "clase social" parte desde el concepto de "clase social". Esta ha sido muy estudiada y discutida por personas especializadas en sociología. Según, Moreno (2008), los primeros planteamientos de ese análisis proceden de Karl Marx y Max Weber ${ }^{1}$. Ahí podemos ver las fuertes contradicciones que se producían alrededor de lo que se establecía entre propiedad del capital y de los medios de producción. Así se derivaba la división de la población (en capital) clase capitalista y clase proletaria. Los que no se ubicaban en ninguna de estas dos se consideraban como residuos de la economía pre capitalista con la tendencia a desaparecer. La división se amplía con el punto de vista de Weber, al afirmar que las clases dependen del capital y que junto a la habilidad y la educación, originan diferentes oportunidades para el individuo como tal. Es por eso que él nos habla de cuatro clases: propietaria, administrativa, pequeños comerciantes y trabajadores. Weber, le brinda una fuerte importancia a la estratificación social a quien considera como un fenómeno multidimensional en el que intervienen tres aspectos esenciales que son: la clase, el estatus y el poder.

Por supuesto que, después de algún tiempo estas teorías son rechazadas por la sociología occidental. Luego surgen las ideas de Hans Gerth y Charles Wright Mills son los que dirigen la atención sobre los estratos sociales y enfocan cuatro, los que ellos denominan dimensiones de la estratificación: ocupación, clase, estatus y poder.

Los interesados en este estudio de la clase social centran su atención sobre diversos factores o dimensiones y no establecen diáfanamente los límites entre uno y otro estrato social; las dificultades que enfrentan estos conceptos siguen en discusión. Sin embargo, existen otros esfuerzos por esclarecer estos puntos y dentro de estos se encuentra la postura norteamericana, quien ha basado su visión en teorías de la estratificación, porque se manifiestan indicadores que se interrelacionan para derivar clases, como: la ocupación.

Si miramos lo más reciente, podemos mencionar a Lavob (1972). El es quien difunde un trabajo enfocado hacia la estratificación. Se basa en un índice socioeconómico en donde combina tres elementos: nivel de instrucción, ocupación y los ingresos familiares. También se menciona a Peter Trudgill (1974) quien realizó un estudio en Norwich (Reino Unido) con seis indicadores: ocupación, nivel de instrucción, ingresos, tipo de vivienda, localidad y ocupación del padre. Esto le facilitó dividir en cinco clases: trabajadora, trabajadora media, trabajadora alta, clase media baja y clase media.

1 Ellos fueron quienes se ocuparon de la estructura que el capitalismo industrial generó durante el siglo XIX. (Moreno, 2008: 52). 
En América, le sigue el de H. López Morales (1983: 27-29), él se enfocó en la variable "nivel socio-cultural" en el estudio de San Juan de Puerto Rico. En su análisis sociolingüístico diferencia cuatro niveles: bajo, medio-bajo, medio y medio-alto y a estos le combina tres parámetros: escolaridad, profesión e ingresos. Mientras tanto, en el español de Caracas se destaca Bentivoglio y Sedano (1993:8-12)., quienes manejan factores como: ocupación, del hablante, ocupación del padre, ocupación de la madre, grado de instrucción, condiciones de alojamiento, ingresos totales e ingresos promedio familiar y los niveles bajo, medio bajo, medio, medio alto y alto. Como bien se ve, la tarea sobre esta y otras variables sociales, es compleja. Con referencia a esto Blas (2008) reflexiona:

El problema surge, por lo tanto, cuando disciplinas como la sociología o la sociolingüística se ven obligadas a establecer la adscripción social de los individuos a partir de determinados parámetros objetivos. Por ello, y a pesar de su empleo continuo en ambas tradiciones, el concepto que nos ocupa no ha dejado de revisarse una y otra vez. (p.210).

De esta manera observamos que las diferentes investigaciones sociolingüísticas han brindado relevancia a los llamados factores y niveles. Esas propuestas multidimensionales son permitidas para llegar a lograr los resultados de una diferenciación entre las personas y descubrir esa distancia en relación a las diferencias sociolingüísticas. No olvidemos que la distribución social de la utilización lingüística determina el papel decisivo de los cambios lingüísticos y que estas están íntimamente relacionadas con las variables sociales, como la "edad" y "sexo".

La variable social clase social, se ha trabajado con la variable nivel socio-cultural, como en el caso de López (1983), en este el nivel (socio-cultural) se aprecia como una variable de post-estratificación; bajo, medio-bajo, medio, medio-alto. Mientras tanto, Bentivoglio y Sedano (1993), citados por Moreno (2008), utilizan siete factores que los llevan a representar cinco niveles socio-económicos: ocupación del hablante, ocupación del padre, ocupación de la madre, grado de instrucción, condiciones de alojamiento, ingresos totales e ingreso promedio familiar y los niveles distinguidos son: bajo, medio bajo, medio, medio alto y alto. Moreno (2008), describe el estudio de Broce y Torres (2002), "Agrupan a sus informantes en tres clases sociales a partir de la combinación de cuatro parámetros (ocupación, ingresos, tipo de vivienda y nivel educativo". (p. 215). También nos habla del estudio de Silva-Corvalán (1979) y Sanicky (1988), quienes han justificado la violenta estratificación sociolingüística a través de la variable (f-). Como se ve, es relevante observar que diferentes estudios sociolingüísticos, como los descritos, usan la palabra nivel socio-cultural o nivel socio-económico para referirse a clase.

Los problemas de la variable social clase social son varios, entre ellos está la cantidad de habitantes que pertenecen a diferentes estratos sociales. Que pueden concernir variadas comunidades, así como el movimiento de clases. También que diversas comunidades poseen una organización social heterogénea entre una y otra, ejemplo sociedades industrializadas. Con relación a esto, Moreno (2008) considera que: "Los factores que configuran la clase social en la praxis sociolingüística varían considerablemente en número y jerarquía, en función de los objetivos concretos de cada investigación". (p. 213). 


\section{La red social}

Con respecto al concepto de "Red Social", Lesley Milroy, citado por Fernández (2008) afirma que: "Es un entramado de relaciones directas entre individuos, que actúa como un mecanismo para intercambiar bienes y servicios, para imponer obligaciones y para otorgar los derechos que corresponden a sus miembros". (p. 57). En este concepto queda planteado que las relaciones entre un individuo y otro son esenciales para obtener algo como un intercambio. Esas relaciones permiten desarrollar grados de densidad y multiplicidad. La densidad, depende del número y relaciones de sus miembros. No obstante, cuando (esas relaciones) entre los miembros de una red son variadas (amistad, vecindad, compañerismo) decimos entonces que estamos frente a redes múltiples y si solo es un vínculo (vecindad), es una red múltiple baja.

Ejemplo de la aplicación de "red social" se observa en Blanco (2004). Esta autora advierte sobre la gran divergencia de criterios alrededor de este concepto, sin embargo resalta que este tipo de análisis se deja ver como una herramienta idónea para solucionar cualquiera de los aspectos polémicos sobre el concepto de "clase social". La red social contribuye a encontrar explicaciones a algunos fenómenos lingüísticos que se encuentran vinculados con ciertos procesos de cambios o refuerzos de normas establecidas. Blanco (2004) también establece que "la densidad y la multiplicidad son excelentes indicadores de la presión de la red sobre el individuo, que lo lleva a adoptar o no las normas y valores de la comunidad" (p.31).

Las variables sociales actúan en correspondencia a los marcadores sociolingüísticos. De acuerdo a Blas (2008) estos son "variables sociales más desarrollados, ya que resultan sensibles tanto a los factores sociales como a los estilísticos, portadores de mayor significación social" (p.136). Entonces estaríamos hablando de que algunos marcadores pasan a ser considerados como normales en algunos grupos sociales, ejemplo en la mayoría de los jóvenes nicaragüenses existe una tendencia mayoritaria a utilizar en su forma de saludarse "¿Qué onda loco?, ¿Pasó loco? y otras. Estas expresiones difícilmente serán escuchadas en la generación adulta.

\section{Nivel de instrucción}

Moreno (2008) especifica que: "Educación, nivel o grado de instrucción, estudios o escolaridad son algunas de las denominaciones que ha recibido la variable que se refiere al tipo de formación académica o de titulación conseguidos por los hablantes, lo que está íntimamente relacionado con la cantidad de años que se ha estado estudiando" (p.60). Mientras que Blas (2008) asegura "Dos de los parámetros que más atención han despertado en el análisis de estratificación sociolectal han sido la profesión y el nivel educativo" (p.224). Y en esto ambos autores coinciden, pues la variable nivel de instrucción se incluye entre los factores que integran a la clase social.

Por ejemplo, López (1983: 25) determina trabajar con escolaridad y la clasifica en (1-8) primaria, (1-4) secundaria, (1-4) universidad y (5+) universidad. En Montes y otros (1998: 20) establecen la variable educación en: analfabetos o primaria, bachillerato hasta cerrera intermedia y universitarios o especialización. Blanco (2004: 78), centró la atención en: el nivel de instrucción inferior (A), nivel medio (B) y nivel superior (C). Por su parte Gil (2006: 26) divide al nivel de instrucción en grado 1: hasta la enseñanza primaria; grado 2: hasta la enseñanza secundaria y grado 3: enseñanza superior. 
En el PRESEEA ${ }^{2}$ se puede apreciar, también, el cuidado con que se describe el nivel de instrucción en: 1. Analfabetos, sin estudios. Enseñanza Primaria (hasta 10-11 años de edad aprox.), 5 años aproximadamente de escolarización;

2. Enseñanza Secundaria (hasta 16-18 años de edad aprox.), 10-12 años aproximadamente de escolarización; 3. Enseñanza Superior (universitaria, técnica superior) (hasta 21-22 años de edad aprox.), 15 años aproximadamente de escolarización. No es posible dejar atrás el trabajo de Rosales (2008:46), esta clasifica a sus informantes en los niveles de primaria incompleta, primaria completa, bachillerato incompleto y bachillerato completo.

Con estos datos establecidos la autora comenta que lo decidió así puesto que: Montes Giraldo recomienda entrevistar a personas con la educación primaria como requisito mínimo. Aunque este no fue un criterio a tomar en cuenta, se evitó entrevistar a personas [sic] con un alto nivel académico para evitar la influencia de la lengua estándar y la ultracorrección que habría alterado, obviamente, los resultados de la investigación. (p. 45). Estas investigaciones han hecho sus mejores esfuerzos por tratar de aplicar una metodología acorde a sus diferentes objetivos y son válidas según sus objetivos propuestos.

En Nicaragua algunos investigadores como Rosales (2008) y Mendoza (2013), han realizado grandes esfuerzos por retomar y coincidir en agrupar el nivel de instrucción ${ }^{3}$ en educación primaria, secundaria y superior. Esto obedece a la realidad y generalidad del nivel o grado de instrucción de los managüenses. ALMA ${ }^{4}$ (2005), en los datos poblacionales de los barrios y demás, carece de información sobre el nivel de instrucción de los capitalinos, sin embargo la experiencia como investigadora y la misma suerte de ser habitante oriunda de esta ciudad, Managua, conceden la seguridad para razonar que son esos tres niveles (primaria, secundaria y superior) son los indicadores principales y precisos a observar en cualquier investigación lingüística a nivel departamental y nacional. Al respecto Moreno (2008) confirma que: "A la vista de las distintas posibilidades que se ofrecen, extraemos como primera consecuencia que la división de niveles de instrucción ha de reflejar la realidad de cada comunidad estudiada". (p.62).

\section{La procedencia}

Es otra de las variables sociales fundamentales para experimentar en cualquier estudio sicolingüístico, así pues, López (1983: .29) sigue el criterio de Cedergren (1973b: 26-27) y divide así: primer grupo: sujetos nacidos en la capital o llegados a ella con menos de seis años de edad; segundo grupo: sujetos llegados a la capital entre 6 y 12 años; tercer grupo: sujetos llegados entre los 13 y 20 años y cuarto grupo: sujetos llegados con 21 años o más. Montes y otros (1998: 20-21) consideró como punto de partida el Plano estratificado de los barrios de Bogotá (1981) y seleccionó 60 barrios: 15 de estrato alto, 25 estrato medio y 20 de estrato bajo. En cuanto a Blanco (2004:78) solamente se centra en informantes de Madrid (M) y Fuera (F). Mientras que Gil (2006: 26) especifica que es un estudio sociolingüístico del fonema /s/ de los hablantes del barrio de Salamanca y que sus resultados son comparados con otros de varias zonas de Madrid:

2 Proyecto para el Estudio Sociolingüístico del Español de España y América.

3 Cabe aclarar que un hablante de educación primaria es aquel que haya concluido su sexto grado o esté estudiando actualmente en cualquiera de los grados. De igual manera el criterio es similar para secundaria y superior. Aunque esto puede variar según los objetivos de determinada investigación. 4 Alcaldía de Managua. 


\section{Francis Mendoza La disciplina que estudia las...}

pp. 1-12

Getafe (Martín Butragueño, 1991 y 1995), Alcalá de Henares (Blanco, 2004) y otras localidades rurales. Por su parte, Rosales (2008: 45) selecciona a hablantes de todos los departamentos de Nicaragua para conformar el Atlas de Nicaragua: nivel fonético. Actualmente Managua concentra la mayor población de Nicaragua, esto es posible por ser la capital y por ofrecer mejores oportunidades de trabajo, estudio, salud y demás aspectos sociales. Acerca de esto Blas (2008) confirma que: "Son muchos los lingüistas que, por razones diversas, prefieren abordar el estudio de las grandes ciudades de forma parcial, mediante el análisis de uno o varios de los barrios que las componen". (p. 69).

Podemos concluir diciendo que todas las variables sociales asumen un papel primordial en el tratamiento del análisis de cualquier variante dialectal. Su aplicación en el campo de la investigación lingüística nos abre nuevas perspectivas de análisis de esos fenómenos del lenguaje y nos ayudan a fundamentar mejor los resultados obtenidos.

La Universidad Nacional Autónoma de Nicaragua, Facultad de Educación e Idiomas, Departamento de Español, Área Lingüística, tiene nuevos retos por alcanzar en cuanto a las investigaciones que se están desarrollando desde la perspectiva sociolingüística a nivel nacional, centroamericano y americano. 


\section{- REFERENCIAS}

Amador, E. y Alvarado, F. (2011). Estudio fonético de los fonemas consonánticos articulados por los hablantes del municipio de Palacagüina, departamento de Madriz, desde una perspectiva sociolingüística durante el periodo de 2011. Tesis de licenciatura en filología y comunicación no publicada, Universidad Nacional Autónoma de Nicaragua, Managua, Nicaragua.

Bentivoglio, P. (2006). La variación sociofonológica. Español Actual, 69:29-42.

Blanco, A. (2004). Estudio sociolingüístico de Alcalá de Henares. Alcalá de Henares: Servicio de Publicaciones de la Universidad de Alcalá.

Blas, J. (2008). Sociolingüística del español. Madrid: Ediciones Cátedra.

Canfiel, L. (1962). La pronunciación del español en América. Bogotá: Instituto Caro y Cuervo.

Cestero, A., Molina, I. y Paredes, F. (2006). Estudios sociolingüísticos del español de España y América. Madrid: Arco/Libros.

Conde, J. (2007). Sociolingüística histórica. Madrid: Gredos.

Dubois, J. y otros. (1994). Diccionario de Lingüística. Madrid: Alianza Editorial.

Fasold, R. (1996). Introducción a la sociolingüística. Madrid: Visor Libros, S. L.

López, H. (1983). Estratificación social del español de San Juan de Puerto Rico. México: Universidad Nacional Autónoma de México.

López, H. (1989). Sociolingüística. Madrid: Gredos.

López, H. (1999). El español de América, América Central. Madrid: Arco, Libros.

Montes, G. (1990). Dialectología y geografía lingüística. Bogotá: Instituto Caro y Cuervo.

Moreno, F. (1990). Metodología sociolingüística. Madrid: Gredos.

Moreno, F. (2008). Principios de sociolingüística y sociología del lenguaje. Barcelona: Ariel, S. A.

Penny, R. (2004). Variación y cambio en español. Madrid: Gredos.

Rosales, M. (2008). Atlas lingüístico de Nicaragua. Nivel Fonético. Análisis geolingüístico pluridimensional. Managua: PAVSA. 